\title{
Desarrollo de zeolitas Sn-ZSM-11 para la síntesis de lactatos de alquilo
}

\section{Development of Sn-ZSM-11 zeolites for the synthesis of alkyl lactates}

Presentación: 10/10/2019

\section{Doctorando:}

\section{Emilce Daniela Galarza}

Centro de Investigación y Tecnología Química (CITeQ), UE CONICET-Universidad Tecnológica Nacional - Facultad Regional Córdoba, Córdoba, Argentina

egalarza@frc.utn.edu.ar, emilce.galarza@gmail.com

\section{Director/es:}

\section{María Soledad Renzini, Clara Saux}

\section{Resumen}

En el presente trabajo se exponen distintas alternativas de incorporación de Sn en la zeolita ZSM-11 (estructura MEL): impregnación por vía húmeda, tratamiento alcalino y síntesis hidrotérmica con estaño como heteroátomo; así como diferente contenido de catión metálico $(\sim 2-10 \%$ p/p). La estructura ZSM-11 se confirmó por DRX para todas las muestras, en tanto que en los materiales impregnados la cristalinidad disminuyó a medida que se incrementó la cantidad del metal incorporado al catalizador. El estudio por TPR señaló la reducción de las especies superficiales vecinas a los enlaces $\mathrm{Sn}-0$ y la reducción de $\mathrm{Sn}^{+4}$ a $\mathrm{Sn}^{+2}$. Por el método BET se analizó el área superficial y se encontró que la misma disminuye al aumentar el contenido de $\mathrm{Sn}$ incorporado por impregnación húmeda y por síntesis hidrotérmica. Mediante FTIR con adsorción de piridina se determinó la cantidad, fuerza y relación de sitios ácidos. Dichos materiales exhibieron un buen desempeño catalítico en la conversión de dihidroxiacetona (DHA) a alquil lactatos de cadena corta, éstos últimos representan solventes verdes prometedores.

Palabras claves: Sn-Zeolita, Síntesis, Síntesis y Caracterización, Acidez de Lewis, Lactatos de alquilo, Dihidroxiacetona.

\begin{abstract}
In the present work are exposed different alternatives of incorporation of Sn in zeolite ZSM-11 (structure MEL): wet impregnation, alkaline treatment and hydrothermal synthesis with tin as heteroatom; as well as different metallic cation content $(\sim 2-10 \% \mathrm{w} / \mathrm{w})$. The ZSM-11 structure was confirmed by XRD for all samples, while in the impregnated materials the crystallinity decreased as the amount of metal incorporated in the catalyst increased. The TPR study indicated the reduction of the surface species neighboring the $\mathrm{Sn}-\mathrm{O}$ bonds and the reduction of $\mathrm{Sn}^{+4}$ to $\mathrm{Sn}^{+2}$. By the BET method the surface area was analyzed and it was found that it decreases with the increase of the Sn content incorporated by wet impregnation and by hydrothermal synthesis. By means of FTIR with pyridine adsorption, the quantity, strength and ratio of acidic sites were determined. The previously synthesized and characterized materials materials exhibited a good catalytic performance in the conversion of dihydroxyacetone (DHA) to short chain alkyl lactates, the latter representing promising green solvents.
\end{abstract}

Keywords: Sn-Zeolite, Synthesis and characterization, Lewis acidity, Alkyl lactate, Dihydroxyacetone.

\section{Introducción}

La catálisis heterogénea es una herramienta de gran importancia para la producción de productos químicos de interés a través de la valorización de materias primas provenientes de biomasa. Las zeolitas han demostrado una gran versatilidad en virtud de sus propiedades redox y ácidas regulables. Tradicionalmente, la acidez de Lewis en zeolitas se origina a partir de la presencia de especies de aluminio extrared, su interacción con la acidez de Brønsted ha 
demostrado ser de crucial importancia. La introducción de metales de transición como el Sn, Ga, Ti y Zr, reconocidos como generadores de acidez de Lewis, en matrices con alto contenido de sílice ha sido lograda a través de distintos métodos (Dapsens, 2015). Los sitios ácidos también pueden ser generados por la sustitución isomórfica del silicio por un metal (por ejemplo, Ti, Zr y Sn) en un marco de sílice pura mediante síntesis hidrotérmica. Este método ha desempeñado un papel primordial pero genera preocupaciones sobre su escalabilidad. Por lo tanto, se han desarrollado recientemente métodos más viables desde el punto de vista industrial basados en la metalización postsintética de zeolitas comerciales, utilizando procedimientos sencillos, secos o húmedos, que implican reactivos no tóxicos y que producen materiales activos (Lari, 2016).

Entre los metales de transición mencionados, las zeolitas que contienen estaño han demostrado propiedades catalíticas muy interesantes; lo cual se ha atribuido principalmente a su hidrofobicidad y cristalinidad. Nos enfocamos, por tanto, en la incorporación de Sn en la estructura tipo ZSM-11 (MEL) mediante impregnación húmeda, que deposita el catión metálico sobre la superficie; síntesis hidrotérmica, incorporando al estaño como heteroátomo dentro de la matriz; y tratamiento alcalino, que desilica la matriz generando espacios para el ingreso del metal de interés. Se determinó la influencia que el procedimiento realizado y el contenido de metal incorporado tienen en la estructura final.

Estos catalizadores se han utilizado en la isomerización y oxidación de azúcares y cetonas (Corma, 2002, 2003; Moliner 2010). En este caso se emplearán particularmente en la isomerización de dihidroxiacetona (DHA) a etil lactatos. La DHA se obtiene de la oxidación de glicerol, principal subproducto de la producción de biodiesel. Los lactatos de alquilo de cadena corta representan solventes verdes prometedores, debido a su fuerte capacidad para disolver compuestos de diversas características químicas. Actualmente utilizado en la industria de plastificantes, herbicidas, fármacos y cosméticos.

\section{Experimental}

\section{Síntesis de los catalizadores}

Las zeolitas microporosas con estructura ZSM-11 se prepararon por síntesis hidrotérmica empleando aluminato de sodio y sílice como fuentes de aluminio y silicio respectivamente, hidróxido de tetrabutilamonio (TBAOH) como agente director de estructura y agua destilada (Chu, 1976). Se dejó cristalizar en autoclave a $140^{\circ} \mathrm{C}$ y presión autogenerada durante 10 días. El material obtenido fue lavado con agua destilada hasta alcanzar pH neutro y secado en estufa. Posteriormente se desorbió en corriente de $\mathrm{N}_{2}$ y temperatura programada hasta $500^{\circ} \mathrm{C}$ durante $8 \mathrm{~h}$ y finalmente se calcinó a la misma temperatura. Para obtener la forma $\mathrm{NH}_{4}$-zeolita se realizó un intercambio con una solución de cloruro de amonio $\left(\mathrm{NH}_{4} \mathrm{Cl}\right) 1 \mathrm{M}$ a $80^{\circ} \mathrm{C}$. Luego se desorbió en corriente de $\mathrm{N}_{2}$ y calcinó con las condiciones antes descriptas para obtener la forma protónica H ZSM 11.

En la impregnación húmeda (WI) se utilizó la forma $\mathrm{NH}_{4}$-ZSM-11. Se incorporó cantidad suficiente de tetracloruro de estaño pentahidratado $\left(\mathrm{SnCl}_{4} .5 \mathrm{H}_{2} \mathrm{O}\right)$ para obtener un porcentaje de 2, 5, 7 y $10 \%$ de $\mathrm{Sn}$. La muestra recuperada se desorbió con nitrógeno y se calcinó a $500^{\circ} \mathrm{C}$ por $8 \mathrm{~h}$. Los catalizadores obtenidos se denominaron WI Sn02, WI Sn05, WI Sn07 y WI Sn10.

La síntesis hidrotérmica (HS) se realizó utilizando tetraetilortosolicato (TEOS), $\mathrm{SnCl}_{4} .5 \mathrm{H}_{2} \mathrm{O}$ y TBAOH como fuentes de silicio, estaño y agente director de estructura respectivamente (Mal, 1995). La mezcla resultante se dejó cristalizar por $48 \mathrm{~h}$ a $140^{\circ} \mathrm{C}$ y presión autogenerada, seguido de una calcinación a $550^{\circ} \mathrm{C}$ durante $5 \mathrm{~h}$. El precursor de estaño se incorporó en cantidad necesaria para obtener una relación en masa del 2 y 5\%. A modo de post-tratamiento, se realizó un intercambio iónico con $\mathrm{NH}_{4} \mathrm{Cl}$ 0,1M a temperatura ambiente por $18 \mathrm{~h}$ consecutivas con una relación $300 \mathrm{ml} \mathrm{de} \mathrm{NH}_{4} \mathrm{Cl}$ $0,1 \mathrm{M}$ por gramo de catalizador. Luego se calcinó el material obtenido nuevamente en las condiciones previamente descriptas. Al material obtenido se lo llamó HS Sn02 y HS Sn05.

El tratamiento alcalino (AT) se efectuó sobre zeolitas Na-ZSM-11 (Lari, 2016) adicionándole una solución de hidróxido de sodio $0,3 \mathrm{M}$ y un volumen adecuado de $\mathrm{SnCl}_{4} .5 \mathrm{H}_{2} \mathrm{O}$ para obtener un $5 \% \mathrm{p} / \mathrm{p}$ de $\mathrm{Sn}$, se agitó durante 30 min a $60{ }^{\circ} \mathrm{C}$ y se inactivó en baño de hielo. Se lavó hasta pH 7 y secó. Al material obtenido se lo sometió al posttratamiento anteriormente detallado.

\section{Caracterización de los catalizadores}

La estructura cristalina de los catalizadores fue determinada por difracción de Rayos X (XRD) en un difractómetro $X$ pert PANanalytical, en un rango de $2 \theta$ entre $5-60^{\circ}$, con un paso de $0.026^{\circ}$, a una velocidad de $5^{\circ} / \mathrm{min}$.

El área superficial de los materiales se determinó por el método Brunauer-Emmet-Teller (BET) en un equipo Pulse Chemisorb de Micromeritics 2700 con absorción de $\mathrm{N}_{2}$ a $77 \mathrm{~K}$ y el volumen de los poros con el método t-plot, con datos de las isotermas de adsorción de $\mathrm{N}_{2}$ en un equipo Micromeritics ASAP 2020. 
El contenido efectivo de incorporación de estaño se analizó por espectroscopía de emisión atómica con Plasma Inductivamente Acoplado (ICP) en un equipo ICP-OPTIMA 2100 DV Perkin Elmer.

El análisis de reducción a temperatura programada (TPR) se midió en un equipo Pulse Chemisorb 2720 Micromeritics; las muestras fueron calentadas a razón de $10^{\circ} \mathrm{C} /$ min desde 200 a $800^{\circ} \mathrm{C}$ en presencia de hidrógeno $(5 \%$ $\mathrm{H}_{2} / \mathrm{N}_{2}$ ). La reducción ocurrida se monitoreó por el consumo de $\mathrm{H}_{2}$.

Los análisis de infrarrojo (FTIR) se realizaron en un espectrómetro Thermo Scientific Nicolet iS10 a los efectos de identificar y cuantificar sitios ácidos del material zeolítico, para tal fin se les adsorbió a las muestras piridina al vacío a temperatura ambiente y desorbió a $400^{\circ} \mathrm{C}$ y $10^{-4}$ Torr, empleando una celda termostatizada con una ventana de $\mathrm{CaF}_{2}$ conectada a una línea de vacío. El número de sitios ácidos de Brönsted y Lewis fue calculado con el área de las bandas de absorción a $1545 \mathrm{~cm}^{-1}$ y $1450-1460 \mathrm{~cm}^{-1}$.

\section{Actividad catalítica}

La reacción de isomerización de DHA (Aldrich 97\%) a alquil lactatos se llevó a cabo en un vial de vidrio de $2 \mathrm{ml}$ a $100^{\circ} \mathrm{C}$ con presión autogenerada por $4 \mathrm{~h}$ con agitación constante. Se utilizó etanol (Cicarelli 99,5\%) como medio de reacción, $16 \mathrm{mg}$ de catalizador y $24 \mathrm{mg}$ de DHA (Dapsens, 2014). El análisis y cuantificación de los productos resultantes se realizó en un Cromatógrafo Perkin Elmer Clarus 500 con detector FID y una columna capilar modelo ZB-1 de $30 \mathrm{~m}$ de longitud y 0,53 $\mathrm{mm}$ de diámetro.

\section{Resultados y discusión}

\section{Caracterización de los catalizadores}

El análisis por XRD confirma la estructura del tipo ZSM-11 para todos los materiales estudiados en el presente trabajo (Figura 1). Comparando los difractogramas con el patrón de difracción del óxido de estaño (IV) $\left(\mathrm{SnO}_{2}\right)$ no se observa la fase cristalina de $\mathrm{SnO}_{2}$. Lo que se puede deber a que las especies $\mathrm{Sn}$ se encuentran formando pequeños dominios de $\mathrm{SnO}_{2}$ no detectados por XRD, aún para contenidos de $\mathrm{SnO}_{2}$ cercanos al $10 \% \mathrm{p} / \mathrm{p}$.

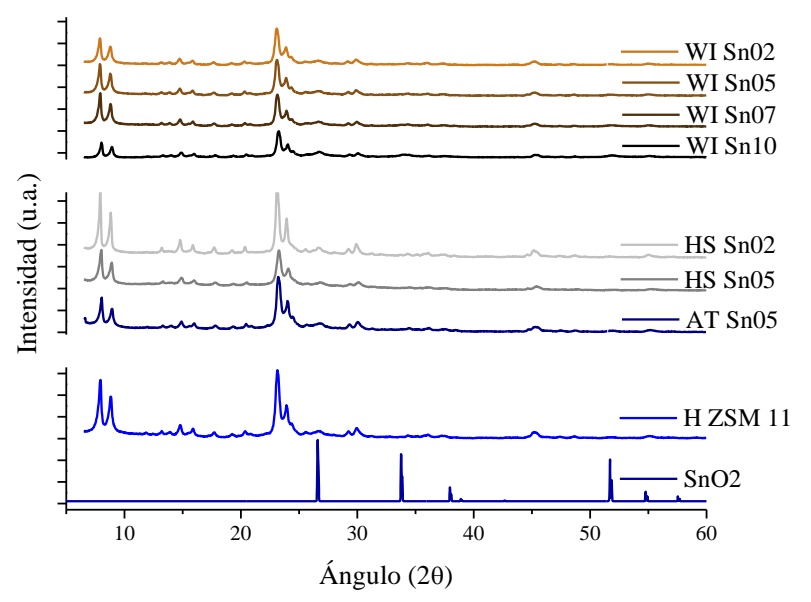

Figura 1. Patrones de XRD de las Sn-ZSM-11

Tabla 1. Caracterización Fisicoquímica

\begin{tabular}{ccccccc}
\hline Catalizador & $\begin{array}{c}\text { Cristalinidad } \\
\text { Relativa }\end{array}$ & $\begin{array}{c}\text { Área } \\
\text { Superficial }\end{array}$ & $\mathbf{V}_{\text {total }}$ & $\mathbf{V}_{\text {micro }}$ & Sn & Si/Al \\
\hline & $\boldsymbol{X} \boldsymbol{R D}$ & $\boldsymbol{B E T}$ & $\boldsymbol{t}$-Plot & $\boldsymbol{t}$-Plot & $\boldsymbol{I C P}$ & $\boldsymbol{I C P}$ \\
\hline H ZSM 11 & 100 & {$\left[\mathbf{m}^{2} / \mathbf{g}\right]$} & {$\left[\mathbf{c m}^{\mathbf{3} / \mathbf{g}]}\right.$} & {$\left[\mathbf{c m}^{3} / \mathbf{g}\right]$} & {$[\boldsymbol{\%}]$} & \\
\hline WI Sn02 & 361 & 0,217 & 0,126 & -- & 23,9 \\
\hline WI Sn05 & 93 & 357 & - & - & 1,4 & 24,8 \\
\hline WI Sn07 & 83 & 363 & - & - & 4,2 & 22,5 \\
\hline WI Sn10 & 56 & 313 & - & - & 7,0 & 22,9 \\
\hline HS Sn02 & 100 & 302 & 0,196 & 0,106 & 10,5 & 23,1 \\
\hline HS Sn05 & 92 & 325 & 0,236 & 0,087 & 4,9 & - \\
\hline AT Sn05 & 100 & 389 & 0,307 & 0,105 & 5,0 & 22,2 \\
\hline
\end{tabular}

El análisis de ICP en los materiales obtenidos comprueba que las cantidades calculadas inicialmente en la preparación de los catalizadores generaron el resultado esperado de incorporación de estaño, cualquiera sea el método utilizado. A su vez, la relación Si/Al no sufrió modificaciones luego de los distintos tratamientos realizados (Tabla 1). 
La Figura 2 muestra los espectros de TPR, todas las muestras presentan perfiles similares que consisten en un hombro alrededor de los $300^{\circ} \mathrm{C}$ y un pico cercano a los $480^{\circ} \mathrm{C}$ estos picos pueden atribuirse a la reducción de las especies superficiales vecinas a los enlaces Sn-0 y a la reducción de $\mathrm{Sn}^{+4}$ a Sn ${ }^{+2}$, respectivamente (Haneda, 2001). La muestra impregnada con un $10 \%$ de Sn presenta también un pico marcado a $680^{\circ} \mathrm{C}$, correspondiente a la reducción de $\mathrm{Sn}^{+2}$ a Sn${ }^{0}$. El corrimiento de los picos hacia menores temperaturas indicaría una menor interacción del metal con la superficie de la zeolita soporte, como el caso de la muestra sometida a tratamiento alcalino.

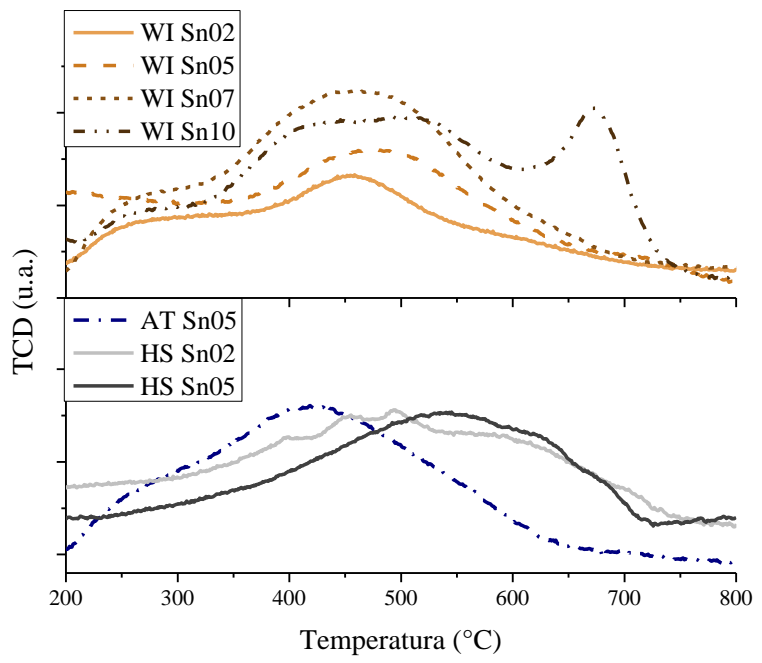

Figura 2. Espectros de Reducción a temperatura Programada

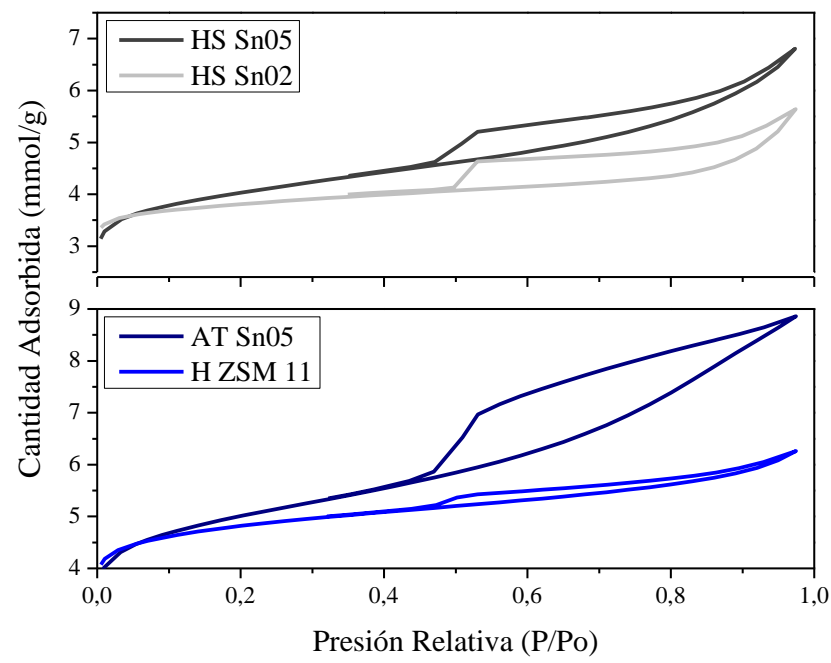

Figura 3. Isotermas de Adsorción de Nitrógeno

Los valores de área superficial BET (Tabla 1) determinadas para las muestras WI y HS resultaron ser menores que las de la matriz zeolítica. Dicho comportamiento puede deberse al bloqueo de poros producido por las especies de $\mathrm{Sn}$ incorporada durante los tratamientos. Para el caso de la muestra sometida a tratamiento alcalino (AT) se puede observar un incremento del valor de área superficial.

Las isotermas de adsorción-desorción de nitrógeno (Figura 3) muestran curvas tipo II, según la clasificación de la IUPAC (Thommes, 2015), característica de adsorbentes microporosos. La forma de la curva es el resultado de adsorción mono- y multicapa sin restricción hasta altos valores de $\mathrm{P} / \mathrm{P}_{0}$. La curvatura gradual a bajos valores de $\mathrm{P} / \mathrm{P}_{0}$ indica la superposición de la cobertura de monocapas y el inicio de la adsorción de múltiples capas. Por otro lado, se observan ciclos de histéresis tipo H4. Este comportamiento es típico de agregados zeolíticos y zeolitas mesoporosas. De este estudio también se observa un aumento del volumen total de poros luego del tratamiento alcalino (muestra AT Sn05) respecto de la matriz protonada (H ZSM 11), confirmando los resultados anteriores.

La naturaleza y fuerza de los sitios ácidos presentes en las muestras se investigó por FTIR de piridina adsorbida a temperatura ambiente, y desorbida a $250^{\circ} \mathrm{C}, 350^{\circ} \mathrm{C}$ y $400^{\circ} \mathrm{C}$.

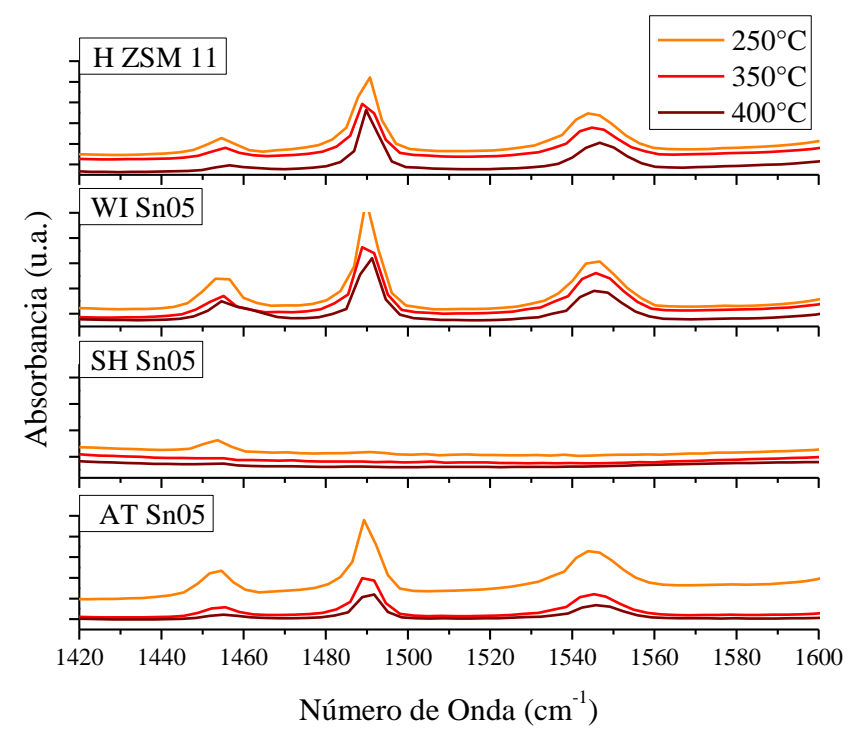

Figura 4. Espectros FTIR de piridina adsorbida a temperaturas crecientes.
Tabla 2. Cuantificación de sitios ácidos

\begin{tabular}{ccc}
\hline Catalizador & $\begin{array}{c}\text { Sitios ácidos } \\
\text { totales }^{\mathbf{a}}\end{array}$ & $\mathbf{L}^{\mathbf{6}} \mathbf{}$ \\
\hline [mm de Py/g] & \\
\hline H ZSM 11 & 82,64 & 0,109 \\
\hline WI Sn02 & 76,19 & 0,236 \\
\hline WI Sn05 & 90,83 & 0,360 \\
\hline WI Sn07 & 98,18 & 0,294 \\
\hline WI Sn10 & 77,90 & 0,299 \\
\hline HS Sn02 & 0,57 & 6,483 \\
\hline HS Sn05 & 0,89 & 6,558 \\
\hline AT Sn05 & 37,35 & 0,135 \\
\hline
\end{tabular}

a Sitios ácidos totales determinados desde el espectro de desorción de piridina a $400^{\circ} \mathrm{C}$,

b L/B (relación sitios ácidos de Lewis y Brönsted). 
En la Figura 4 se presentaron los espectros de las muestras con un contenido de estaño del 5\% y de la matriz protonada a modo de comparación. La evolución de los sitios con la temperatura da cuenta de la fuerza de los mismos. El pico a $1445 \mathrm{~cm}^{-1}$ se asocia a los sitios de Lewis, y el de $1545 \mathrm{~cm}^{-1}$ con la acidez de Brönsted, siendo esta última superior en todos los casos salvo para las muestras obtenidas por síntesis hidrotérmica. En la Tabla 2 se presenta la cuantificación de sitios ácidos totales y la relación entre sitios ácidos de Lewis y Brönsted.

\section{Isomerización de DHA a etil lactato}

Para el estudio de la obtención de etil lactato a partir de la isomerización catalítica de DHA, con alcohol etílico como solvente, se analizó el efecto del método de incorporación de estaño así como el porcentaje de catión efectivamente incorporado en la matriz zeolítica en la conversión del reactivo.

El mecanismo de reacción que conduce a la generación de EL y a los otros productos, se presenta en el Esquema 1. Las etapas involucradas consisten en primera instancia en la isomerización de DHA al gliceraldehído (GLA), con las condiciones experimentales utilizadas el equilibrio se vería desplazado hacia la DHA (Dapsens, 2014). La etapa siguiente consiste en la deshidratación de la DHA y GLA para dar aldehído pirúvico (PA). El PA puede luego convertirse por incorporación de una molécula de etanol en lactato de etilo (EL), o en hemiacetal del aldehído pirúvico (PAEH) y dietilacetal del aldehído pirúvico (PADA). Posteriormente el PAEH por isomerización puede generar EL.

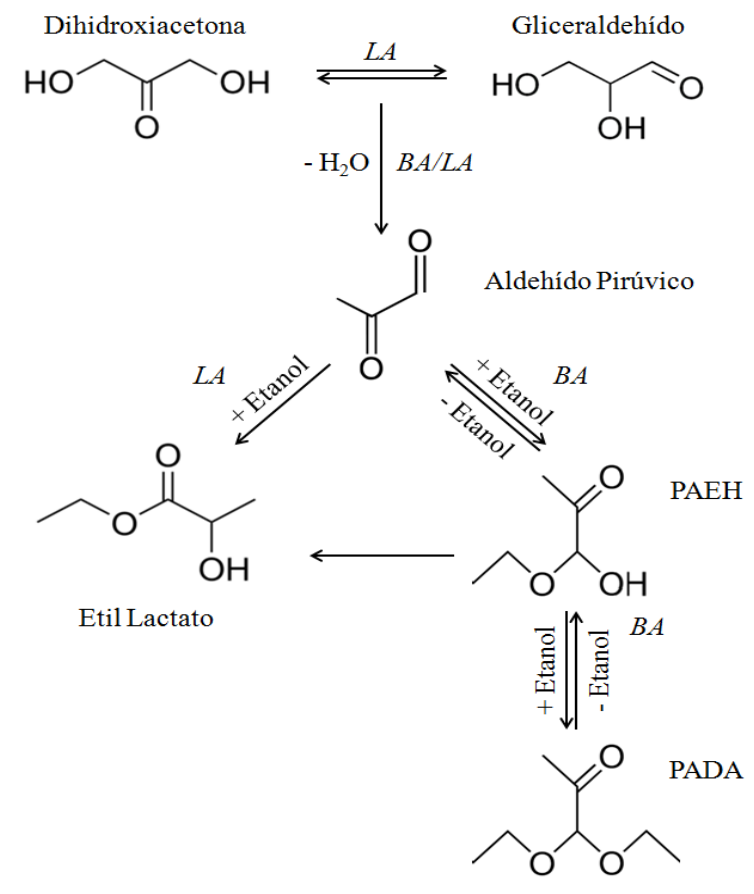

Esquema 1. Mecanismo de reacción

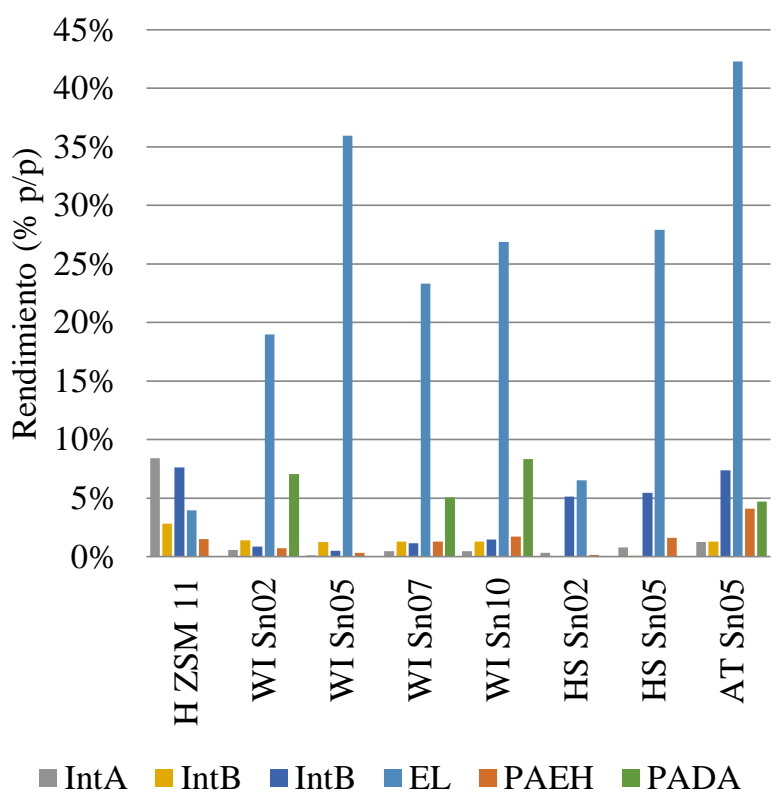

Figura 5. Rendimientos a los productos principales obtenidos.

En la Figura 5 se observan los rendimientos a los productos principales obtenidos, así como algunos intermedios (aún no identificados) IntA, IntB y IntC. Los porcentajes de rendimientos a los productos e intermediarios empleando la zeolita H ZSM 11 son menores al 10\% p/p. Mientras que al emplear las versiones modificadas con Sn los rendimientos se han visto incrementados, excepto para la muestra HS Sn02, este resultado puede adjudicarse al menor valor de área superficial.

Se observa un comportamiento distinto de acuerdo al tipo de incorporación de estaño. Para el caso de zeolitas impregnadas el rendimiento mayoritario a EL se presenta al emplear la muestra WI Sn05 (cercano al 40 \%p/p). Esta carga de Sn se confirma como óptima en los materiales obtenidos por síntesis hidrotérmica. El material expuesto a tratamiento alcalino presenta el mejor rendimiento con valor superior al 50\% p/p.

El tipo de sitio ácido, Brönsted o Lewis, juega un rol fundamental en la obtención de EL y/o otros productos oxigenados. Así es que los sitios ácidos de naturaleza Lewis favorecen la producción de EL como producto final, mientras que los de Brönsted conducen a la generación de PAEH. Lo mencionado anteriormente se condice con los resultados de actividad catalítica donde se observan rendimientos mayoritarios a EL al emplear las zeolitas WI, HS y AT con una carga de 5\%p/p de Sn, que son las muestras con mayor relación L/B. Mientras que para la muestra sometida a tratamiento alcalino (AT Sn05) el mayor rendimiento obtenido al producto deseado y a PAEH se debería a un efecto sinérgico entre acidez de Bronsted y Lewis, el mayor valor de área superficial y volumen total de poro.

Se infiere entonces que la isomerización de DHA hacia el producto de interés estaría favorecida por a la presencia de estaño, la cristalinidad de las matrices, el tipo de acidez y el tamaño de los poros de la matriz. 


\section{Conclusiones}

Las zeolitas modificadas con Sn se prepararon por impregnación por humedad incipiente, síntesis hidrotérmica y tratamiento alcalino. Los materiales preparados presentaron mayores relaciones L/B que la zeolita H ZSM 11; promoviendo la conversión de DHA y etanol en EL en condiciones suevas de reacción.

La caracterización de los materiales confirmó la estructura tipo ZSM 11 para todas las muestras la cantidad efectiva de estaño incorporado. Las isotermas de adsorción-desorción de nitrógeno muestran curvas características de materiales microporosos, por otro lado, se observan ciclos de histéresis típicos zeolitas mesoporosas en los materiales sometidos a tratamiento alcalino y síntesis hidrotérmica. De este estudio también se observa un aumento del volumen total de poros de la muestra AT Sn05 respecto de la matriz protonada, confirmando los resultados anteriores.

Los mayores rendimientos a lactato de etilo, se obtuvieron cuando se emplearon Sn-ZSM-11 con una carga del 5\%p/p del catión metálico. La reacción que fue catalizada por el material obtenido por tratamiento alcalino exhibió un rendimiento superior al $50 \% \mathrm{p} / \mathrm{p}$.

\section{Referencias}

Chu, P. U.S. Patent No. 3,709,979. Washington, DC: U.S. Patent and Trademark Office. 9 Jan. 1973.

Corma, A., Domine, M. E., Nemeth, L., \& Valencia, S. (2002). Al-free Sn-beta zeolite as a catalyst for the selective reduction of carbonyl compounds (Meerwein- Ponndorf-Verley reaction). Journal of the American Chemical Society, 124(13), 3194-3195. doi.org/10.1021/ja012297m.

Corma, A., Domine, M. E., \& Valencia, S. (2003). Water-resistant solid Lewis acid catalysts: Meerwein-Ponndorf-Verley and Oppenauer reactions catalyzed by tin-beta zeolite. Journal of Catalysis, 215(2), 294-304. doi.org/10.1016/S00219517(03)00014-9.

Dapsens, P. Y., Kusema, B. T., Mondelli, C., \& Pérez-Ramírez, J. (2014). Gallium-modified zeolites for the selective conversion of bio-based dihydroxyacetone into C1-C4 alkyl lactates. Journal of Molecular Catalysis A: Chemical, 388, 141-147. doi.org/10.1016/j.molcata.2013.09.032.

Dapsens, P. Y., Mondelli, C., \& Pérez-Ramírez, J. (2015). Design of Lewis-acid centres in zeolitic matrices for the conversion of renewables. Chemical Society Reviews, 44(20), 7025-7043. DOI:10.1039/C5CS00028A.

Haneda, M., Ohzu, S. I., Kintaichi, Y., Shimizu, K. I., Shibata, J., Yoshida, H., \& Hamada, H. (2001). Sol-Gel Prepared SnAl203 Catalysts for the Selective Reduction of NO with Propene. Bulletin of the Chemical Society of Japan, 74(11), 2075-2081. doi.org/10.1246/bcsj.74.2075.

Lari, G. M., Dapsens, P. Y., Scholz, D., Mitchell, S., Mondelli, C., \& Pérez-Ramírez, J. (2016). Deactivation mechanisms of tin-zeolites in biomass conversions. Green Chemistry, 18(5), 1249-1260. DOI:10.1039/C5GC02147B.

Mal, N. K., Ramaswamy, V., Ganapathy, S., \& Ramaswamy, A. V. (1995). Synthesis of tin-silicalite molecular sieves with MEL structure and their catalytic activity in oxidation reactions. Applied Catalysis A: General, 125(2), 233-245. doi.org/10.1016/0926-860X(95)00021-6.

Moliner, M., Román-Leshkov, Y., \& Davis, M. E. (2010). Tin-containing zeolites are highly active catalysts for the isomerization of glucose in water. Proceedings of the National Academy of Sciences, 107(14), 6164-6168. doi.org/10.1073/pnas.1002358107.

Thommes, M., Kaneko, K., Neimark, A. V., Olivier, J. P., Rodriguez-Reinoso, F., Rouquerol, J., \& Sing, K. S. (2015). Physisorption of gases, with special reference to the evaluation of surface area and pore size distribution (IUPAC Technical Report). Pure and Applied Chemistry, 87(9-10), 1051-1069. doi.org/10.1515/pac-2014-1117. 